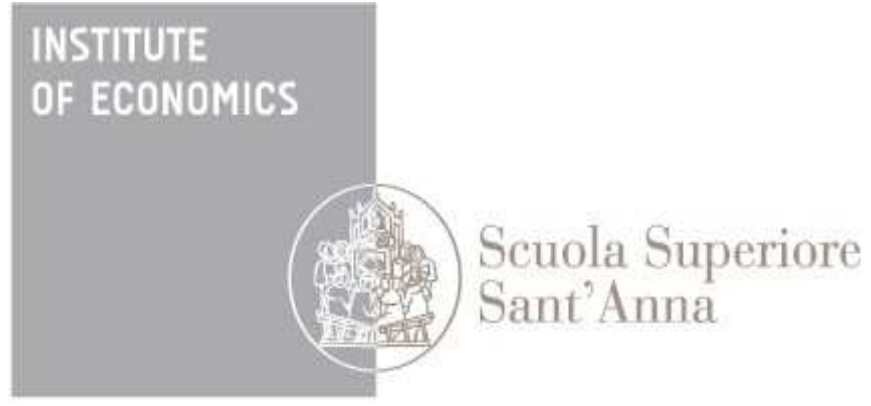

LEM | Laboratory of Economics and Management

Institute of Economics

Scuola Superiore Sant'Anna

Piazza Martiri della Libertà, 33 - 56127 Pisa, Italy ph. +3905088.33 .43$

institute.economics@sssup.it

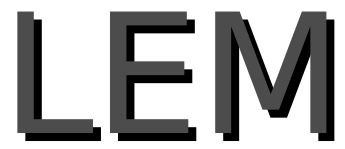

Working Paper Series

\title{
Dynamic Increasing Returns and Innovation Diffusion: bringing Polya Urn processes to the empirical data
}

\author{
Giovanni Dosi ${ }^{\circ}$ \\ Alessio Moneta ${ }^{\circ}$ \\ Elena Stepanova ${ }^{\circ}$
}

'Institute of Economics, Scuola Superiore Sant'Anna, Pisa, Italy 


\title{
Dynamic Increasing Returns and Innovation Diffusion: bringing Polya Urn processes to the empirical data
}

\author{
Giovanni Dosi $^{1} \quad$ Alessio Moneta $^{1} \quad$ Elena StePanova $^{1 *}$
}

\begin{abstract}
The patterns of innovation diffusion are well approximated by the logistic curves. This is the robust empirical fact confirmed by many studies in innovations dynamics. Here we show that the logistic pattern of innovation diffusion can be replicated by the time-dependent stochastic process with positive feedbacks along the diffusion trajectory. The dynamic increasing returns process is modeled by generalized Polya urns. So far, urn models have been mostly used to study the [path-dependent] limit properties. On the contrary, this work focuses on the transient [finite time] properties studying the conditions under which urn models capture the logistic trajectories which often track empirical diffusion process. As examples, we calibrate the process to match several cases of diffusion of motor ships in European countries.
\end{abstract}

Keywords: Polya urn schemes, Innovation diffusion, Logistic diffusion pattern, Dynamic increasing returns. JEL Classification: C63, O33

\footnotetext{
${ }^{1}$ Scuola Superiore Sant'Anna, Institute of Economics, Piazza Martiri della Libertà 33, 56127 Pisa, Italy. Emails: giovanni.dosi@santannapisa.it, alessio.moneta@santannapisa.it,elena.stepanova@santannapisa.it

*Acknowledgements: The support from the European Union's Horizon 2020 Research and Innovation Programme under grant agreement No. 649186 - ISIGrowth is gratefully acknowledged
} 


\section{Introduction}

The logistic pattern of innovation diffusion is one of the most robust empirical regularities in the literature on adoptions of new technologies. Usually firms or consumers adopt new product sequentially, and the vast majority of eventual users adopt well after the early introduction of a new product. So the diffusion process takes off rather slowly, then, after a while, it accelerates, and finally, it slows down until a proximate saturation level is approached. Most empirical studies have shown that observed diffusion patterns are well approximated by logistic curves. This empirical regularity holds for intra-firm, intra-industry, economywide adoptions and both for production processes and new products: within a large literature see the early examples from Griliches (1957) and Mansfield (1961), all the way to Comin et al. (2006) and Nuvolari et al. (2011). However the precise form of logistic diffusion pattern varies considerably across technologies. Moreover, some technologies do not take off at all.

The fundamental interpretative question concerns the determinants of particular diffusion paths. Not surprisingly, the economic discipline has produced different and partly conflicting theoretical explanations (for overviews see Davies, 1979; Dosi, 2000; Stoneman and Battisti, 2010). There are many divides in the proposed interpretations of time-consuming diffusion processes which relate essentially to (i) the degrees of heterogeneity of potential adopters, (ii) their "rationality", (iii) the nature of "returns to adoption", (iv) the postulated collective equilibrium (or not) outcomes of adoption decisions.

A distinct family of models of diffusion, based on Polya urn process, first, build on the evidence that agents are heterogeneous on whatever dimension one may choose, including their beliefs, expectations and degree of "rationality", whatever that means in evolving worlds. Second, they assume dynamic increasing returns of some kind, for example due to network externalities, learning curve interactions between demand and supply, imitation effects, etc. Third, they dispose of any notion of "equilibrium" diffusion processes, at the same time so far they have been limiting themselves to the asymptotic properties of such processes. However, by doing that, the major drawback has been the decoupling between the model conclusions and empirical observations. The former are limit properties while the latter are inevitable transients in our finite worlds. In many circumstances predictions on the limit are also good interpretation on what finally happens on diffusion (does $x$ or $y$ diffuse at the end?). Nonetheless such models have been so far largely silent on the patterns of the diffusion process itself. Here we try to bridge the gap.

Polya urns are formal instrument particularly suited for path-dependent stochastic process. They are natural candidate to interpret processes of competition among alternative technologies, but also, more metaphorically, competing "visions of the world", or competing "organizational forms". Needless to say, they are in tune with evolutionary ideas in economics (e.g. Arthur et al., 1987; Bassanini and Dosi, 2006; Bottazzi and Secchi, 2003; Dosi et al., 2015; Dosi and Kaniovski, 1994; Dosi et al., 1994; Windrum, 2004), and also in other disciplines, such as sociology, biology and medicine (e.g. Donnelly, 1986; Mahoney, 2000; Zhu and $\mathrm{Hu}, 2012$ ).

Of course, diffusion dynamics with heterogeneous agents and dynamic increasing return imply that history counts. Several outcomes are possible and the cumulation of micro decisions "selects" one of the possible long-term outcomes. In other words, the past shapes, in probability, the future, and this effect self-reinforces along the time-path trajectory. The urn-process with positive feedback asymptotically 
converges to one of the possible outcomes. This asymptotic property allows to explain the effect of "lockin" into one of technologies [not necessarily a superior one] available on the market with increasing returns to adoption. However, we all live in a finite time and we are also interested in understanding the finite time behavior of the process. So far, one has studied at the most the determinants of different speeds of convergence (Bassanini and Dosi, 2006). The present paper focuses indeed on the transient properties of Polya Urn diffusion processes. Here we show that the trajectory leading to the "lock-in" into one of available technologies tends to display a logistic shape, thus reproducing empirically observed trajectories of technology diffusion. We calibrate the model on some empirical examples of motor ships diffusion in Europe. To our knowledge this is the first and quite promising attempt to qualitatively match an evolutionary model based on Polya urn with empirical time-series.

In the next section we briefly present the Polya urn process. In section 3 we describe the empirical data on the diffusion of motor ships. Section 4 calibrates the Polya urn process on the empirical data.

\section{Polya Urn stochastic process}

Generalized Urn Schemes formalize non-stationary Markov chains with a growing number of states. In the context of technologies diffusion, the stochastic process concerns market share of a particular technology, $x_{t}$. It lives on a one-dimensional simplex $(0,1)$, capturing the shares of, for simplicity, two technologies. For our purposes here let us consider two types of urn schemes, namely, additions to the urn (Kaniovski and Pflug, 1995; Hill et al., 1980; Arthur et al., 1986, 1987) and withdrawals from the urn (Panholzer and Kuba, 2012; Kuba and Panholzer, 2012; Siegrist, 1987).

Additions to the urn

Consider an urn of infinite capacity with balls of two types, $\mathbf{A}$ and $\mathbf{B}$. For us $\mathbf{A}$ and $\mathbf{B}$ will represent two competing technologies. $x_{k}$ is the share of $\mathbf{A}$-balls in the urn at the step $k$. Starting with $n_{A} \geq 1 \mathbf{A}$-balls and $n_{B} \geq 1 \mathrm{~B}$-balls in the urn, a ball is added at steps $k=1,2, \ldots$, i.e. a new adopter chooses one of the two technologies. It will be $\mathbf{A}$ with the probability $p(x)$ and $\mathbf{B}$ with the probability $1-p(x)$. The corresponding random variable is $\xi\left(x_{k}\right)$ has Bernoulli distribution:

$$
\xi\left(x_{k}\right)= \begin{cases}1 & \text { with probability } \quad p\left(x_{k}\right) \\ 0 & \text { with probability } 1-p\left(x_{k}\right)\end{cases}
$$

The probability function $p(x)$ maps the share of A-balls in the urn into the probability of its adoption, i.e. $\mathbb{R}(0,1)$ into $\mathbb{R}[0,1]$ (for example, Figure 1 ). Depending on the form of the probability function $p(x)$ the urn-process will exhibit different dynamics over time, formally given by the relation:

$$
x_{k+1}=\frac{n_{A}+\sum_{i=1}^{k} \xi\left(x_{i}\right)}{n_{A}+n_{B}+k}, \text { for } k \geq 1 \text { and } x_{1}=\frac{n_{A}}{n_{A}+n_{B}}
$$

Denote $\xi\left(x_{k}\right)-\mathbf{E}\left(\xi\left(x_{k}\right)\right)=\xi\left(x_{k}\right)-p\left(x_{k}\right)$ by $\lambda\left(x_{k}\right)$, and note that $\mathbf{E}\left(\lambda\left(x_{k}\right)\right)=0$. Then [2] can be rewritten as 


$$
x_{k+1}=x_{k}+\frac{p\left(x_{k}\right)-x_{k}}{n_{A}+n_{B}+k}+\lambda\left(x_{k}\right), \text { for } k \geq 1 \text { and } x_{1}=\frac{n_{A}}{n_{A}+n_{B}}
$$

The system [3] shifts on average at each step $k \geq 1$ on the value $\frac{p\left(x_{k}\right)-x_{k}}{n_{A}+n_{B}+k}$.

Consequently limit states of the sequences $\left\{x_{k}\right\}$ have to belong to the set of zeros of the function $p\left(x_{k}\right)-x_{k}$ (for $x_{k} \in[0,1]$ ). Limit state $\theta$ is called stable if for every small enough $e_{1}>0, e_{2}>0$

$$
(p(x)-x)(x-\theta)<\delta\left(e_{1}, e_{2}\right)<0
$$

given that $e_{1} \leq|x-\theta| \leq e_{2}$ for $x \in[0,1]$. The set of stable limit states $\Theta$ of the urn process depends on the form of the probability function $p(x)$. And the convergence rate to $\theta \in \Theta$ depends upon the smoothness of $p(x)$. Limit properties of the process have been extensively studied in Hill et al. (1980), Arthur et al. (1986) Kaniovski and Pflug (1995) and Dosi and Kaniovski (1994).

Withdrawals from the urn

Consider the foregoing urn with balls of two types, $\mathbf{A}$ and $\mathbf{B}, n_{A} \geq 1$ and $n_{B} \geq 1$, and with $x_{k}$ being the share of $\mathbf{A}$-balls in the urn at the step $k$. A ball is removed from the urn at steps $k=1,2, \ldots$ For us it will represent the scraping of old equipment by users. The instrument item embodying technology A will be scrapped with probability $1-p\left(x_{k}\right)$ and the item embodying technology $\mathbf{B}-$ with probability $p\left(x_{k}\right)$. The corresponding random variable $\varepsilon\left(x_{k}\right)$ has Bernoulli distribution:

$$
\varepsilon\left(x_{k}\right)=\left\{\begin{array}{lll}
1 & \text { with probability } 1-p\left(x_{k}\right) \\
0 & \text { with probability } \quad p\left(x_{k}\right)
\end{array}\right.
$$

Note the reciprocity of Bernoulli distributions for the case of adoption of the technology $\mathbf{A}, \xi\left(x_{k}\right)[1]$ and for the case of abolishment of the technology $\mathbf{A}, \varepsilon\left(x_{k}\right)$ [4].

The dynamics in time is given by the same type of the relation as in [2] but with the opposite sign in front of $\varepsilon\left(x_{i}\right)$ and $k$ because balls are removed from the urn:

$$
x_{k+1}=\frac{n_{A}-\sum_{i=1}^{k} \varepsilon\left(x_{i}\right)}{n_{A}+n_{B}-k} .
$$

For simplicity, we assume that both processes, additions to the urn and withdrawals from the urn, have the same probability function $p(x)$. We choose such $p(x)$ that exhibits the increasing returns to the adoption of technology, that has the biggest market share at the time of the adoption, and symmetrically to the scraping of technology that has the smaller market share.

In the following, we study the property of increasing return world. The urn process with the probability function $p(x)$ that embodies increasing returns ${ }^{1}$ has a 'lock-in' into monopoly as the stable limit state.

\footnotetext{
${ }^{1}$ Urn processes with the probability function $p(x)$ that embodies increasing returns are sometimes called time-dependent stochastic processes with positive feedback.
} 
Stable limit states are only 1 and 0 , which correspond to the monopoly of the technology $\mathbf{A}$ or the technology $\mathbf{B}$ respectively.

As an illustration of a possible dynamic mechanism, suppose that potential adopters are only imperfectly informed about the utility of two technologies (A and $\mathbf{B}$ ) available. So they make their choices by asking an odd number $s$ of adopters who have already adopted one of the two technologies and they adopt the technology that the majority of their sample is using. An alternative hypothesis leading to the same effect is that there are positive externalities in adoption which change the returns to the user along the diffusion process, and adopters are influenced by different numbers of users. Hence, the probability function $p(x)$ has the form

$$
p\left(s, x_{k}\right)=\sum_{i=\frac{s+1}{2}}^{s} \mathbb{C}_{s}^{i} x_{k}^{i}\left(1-x_{k}\right)^{1-i},
$$

where $\mathbb{C}_{s}^{i}$ is the number of $i$-combinations from $s$.

For example, suppose $s=3$. So one samples 3 balls from the urn. If 2 of the balls are A-balls, in the process additions to the urn, one will add one more A-ball to the urn; while in the process withdrawals from the urn, one will remove one B-ball from the urn. Dosi and Kaniovski (1994) prove that the probability function [6] exhibits increasing returns [the probability of adoption increases with the market share of the technology, $\left.x_{t}\right]$ and leads to the 'lock-in' into monopoly. The probability function [6] is plotted on Figure 1.

Figure 2(a,b) presents 20 realizations of the process with initially equal market shares of both technologies $(\mathbf{A}$ and $\mathbf{B})-$

(a) 1000 sequential additions to the urn,

(b) 1000 sequential withdrawals from the urn.

As can be seen, technologies have equal chances to be selected as the winning technology by the timedependent process - an asymptotic property of such urn model setup that was widely exploited in evolutionary economics.

Figure 1: Probability function $p(x)$ of the Polya urn stochastic process

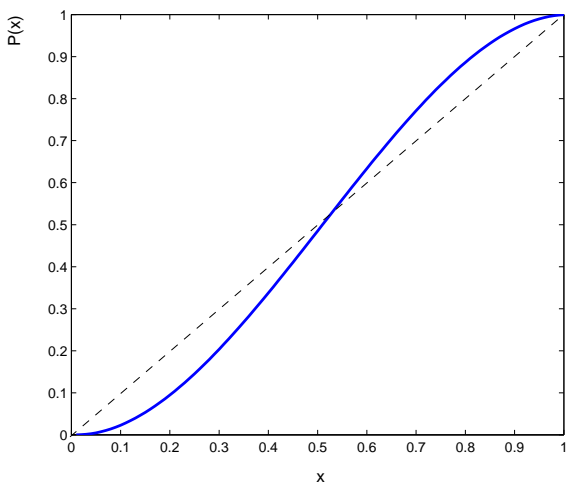

The set of possible limit states of the process is the set $p(x)=x$, i.e. all points of the probability function that lay on the $45^{\circ}$-degree line, namely $0,0.5$ and 1 . Stable limit states are only 0 and 1. 
Figure 2: Realizations of the Polya urn stochastic process

(a) addition to the urn

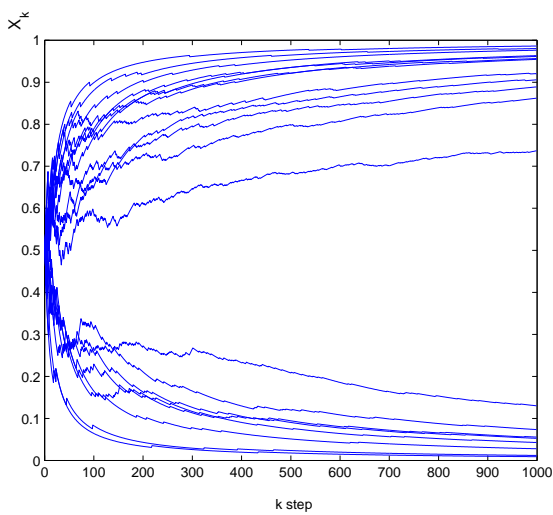

1000 additions to the urn (urn initial size is 10 balls) (b) withdrawal from the urn

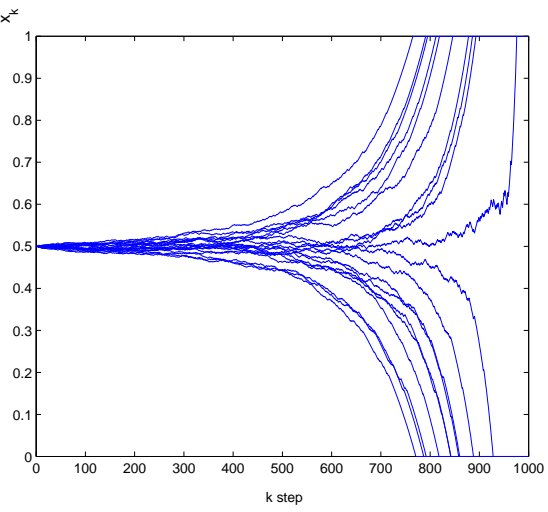

1000 withdrawals from the urn (urn initial size is 1000 balls)

To bring Polya Urn process to the empirical data we need to assume some form of the correspondence between real time [measured in years $t=1,2, .$.$] and steps with which the urn process evolves k=1,2, \ldots$. Suppose $n_{t}$ additions to the urn and $m_{t}$ withdrawals from the urn happen in year $t . X_{t}$ is the share of A-balls in the urn in year $t$, and, analogously to [2] and [5], its dynamics can be formalized in the following equation:

$$
X_{T+1}=\frac{n_{A}+\sum_{t=1}^{T}\left(\sum_{i=1}^{n_{t}} \xi_{i}\left(X_{t}\right)-\sum_{j=1}^{m_{t}} \varepsilon_{j}\left(X_{t}\right)\right)}{n_{A}+n_{B}+\sum_{t=1}^{T}\left(n_{t}-m_{t}\right)}
$$

Figure 3 presents realizations of the process [7] with initially equal market shares of both technologies (A and B). $n_{t}$ is modeled as an arithmetical progression with $n_{1}=10$ and step 10, and $m_{t}-$ as an arithmetical progression with $m_{1}=1$ and step 1 . We limit the real time to 40 years and display only realizations that converged to one of the two possible stable limit states within the given time period. Dotted lines are averages across realizations, and tend to display logistic shape.

In the next sections we describe empirical data on the technology diffusion that we have, and we calibrate the urn-process [7] to fit our empirical data.

\section{Increasing returns and the diffusion of motor ships}

Historical examples of increasing returns and "path dependence" basically driven by self-reinforcing processes are growing in number, examples in which existing investments and infrastructure give an increasing advantage to doing things in a certain way, even if the economy could do them differently and possibly better if only one would start from scratch. David (1985) vividly illustrates the ascent to dominance of the 
Figure 3: Realizations of the process [7]

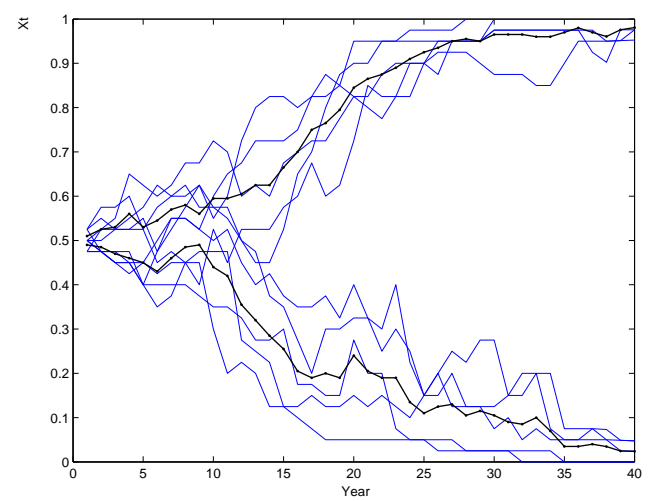

Urn initial size is 40 balls. The number of balls to be added each year is an arithmetical progression with $n_{1}=10$ and step 10. The number of balls to be withdrawn each year is an arithmetical progression with $m_{1}=1$ and step 1 . Only realizations that converged to stable limit states in 40 years time period are plotted. Dotted lines are averages across realizations that converged to one of the two possible stable limit states.

QWERTY keyboard well beyond the period of its purported efficiency. In a similar vein, Arthur (1989) argues that as late as 1914 steam was at least as viable as crude oil for powering vehicles - but the growing influence of the oil industry ensured that much more money went into improving the internal combustion engine than the steam engine. That, plus "small events" (the outbreak of hoof and mouth disease among horses used to refill steam boilers (Cowan, 1990)) induced a snowball effect in favor of internal combustion engines.

The example we consider here is somewhat similar. We have historical time series data on the adoption of motor ships, i.e. ships propelled by an internal combustion engine, usually a diesel engine, in several European countries. Two first diesel-powered ships were launched in 1903, both for river and canal operations: "La Petite-Pierre" in France, powered by Dyckhoff-built diesels, and "Vandal" tanker in Russia, powered by Swedish-built diesels with an electrical transmission. In 1910 the Norwegian built "Fram" - the sailing ship fitted with an auxiliary diesel engine, the first ocean-going ship with a diesel engine. And in 1912 the Danish built the first ocean-going ship exclusively powered by a diesel engine, MS Se-landia (Stapersma, 1996).

In 1925, when steam was absolutely dominating the market of merchant ships, Popular Mechanics Magazine writes: "Commercially, the advantages of motor ship are very great. Boats driven by Diesel motors save $75 \%$ on space needed to carry fuel, this way more space is made available for cargo, with appreciable effect on the earning power of the ship. The fuel bill of a motor ship is small compared with that of a steamship: a motor ship of average size will consume 15 tons of fuel a day, where an oil-burning steamer would need 30 tons. A motor ship can carry $20 \%$ more cargo than a steamship of the same size, because a vast amount of space in the engine room of a steamship is taken up by boilers, which are not needed by the motor ship. Water-ballast tanks, holding a non-paying element on the steamship, are absent from the motor ship" (Wilkins, 1925). It was only after World War One that Diesel's invention began to 
slowly realize its commercial potential. And by 1939 only a quarter of global sea trade was fueled by diesel. But it shouldn't be surprising. As Edwin Mansfield correctly notes in his seminal contribution (Mansfield, 1961), when the innovation replaces equipment that is durable, adopters are reluctant to scrap equipment embodying old technology. In such cases, there is a good chance that a firm's old equipment still has a relatively long useful life. Although rational economic calculation might indicate that replacement would be profitable, firms may be reluctant to scrap equipment that is not fully written off and that will continue to serve for many years.

Even if a motor-engine was improved enough to become a superior technology in comparison with a steam-engine, the adoption of it didn't happen immediately. Figure 4 shows the diffusion of motor ships in five European countries. As can be seen from this Figure, it took from 18 (in Norway) to 8 (in Finland) years for a motor technology to arrive from $25 \%$ of the market share to $50 \%$. The purchase price advantage and the existing experience of exploitation of the old technology [as well as developed market of the spare parts and repair services] made users persistent in preferring it to the new technology. On the other hand, the new technology represented attractive cost saving opportunity. So, when buying new ship, an adopter had to make a choice between the old and the new technology balancing their preferences towards advantages and disadvantages of the two, influenced also by its incumbent capabilities, the observation of its competitors, the availability of supporting infrastructures, etc.

Figure 4: Percentage of motor ships in the merchant fleet of European countries

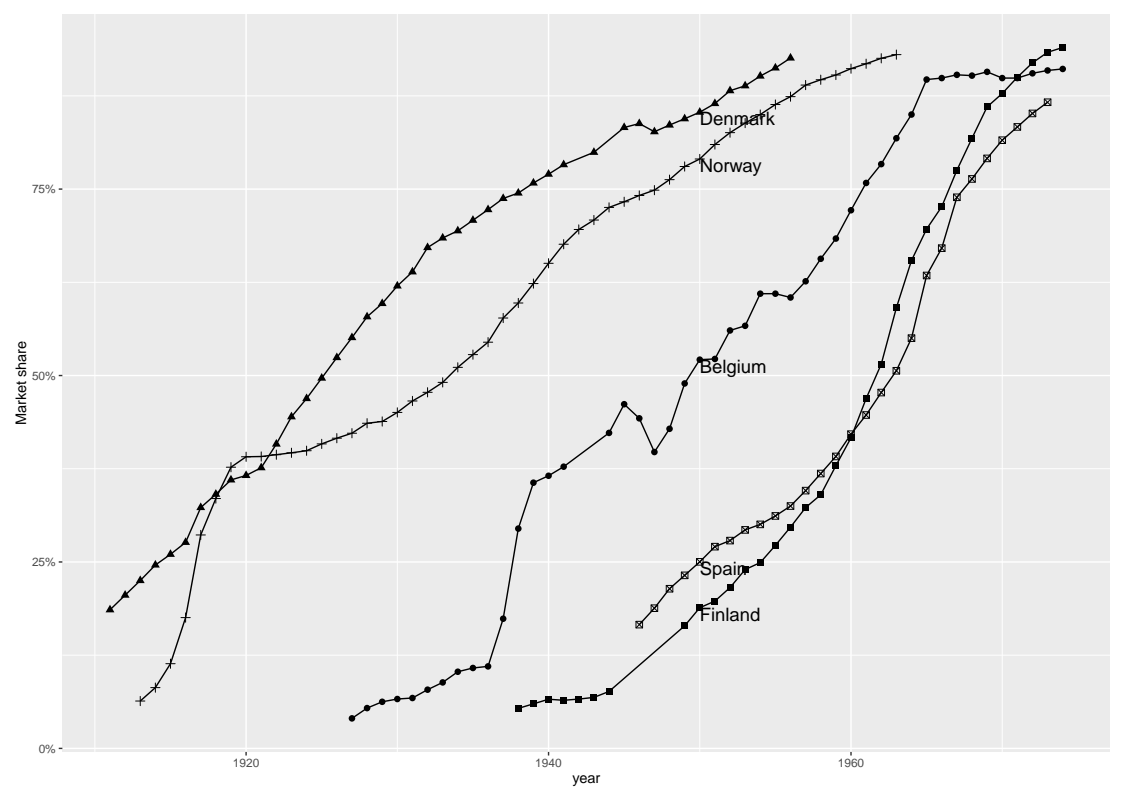

Source: Mitchell (1998) 


\section{Calibration of the stochastic process}

Annual time series on the number of motor ships in the merchant fleet and the total size of the merchant fleet is taken from Mitchell (1998). For the calibration of our model we will use the data of five countries: Belgium, Finland, Spain, Norway and Denmark, for which the time series are most complete (Figure 4).

We suppose that initial chances of adoption of one of the technologies, motor or steam, were equal. ${ }^{2}$ This is quite plausible assumption to make. As we argued above, there are many reasons why firms were reluctant to adopt the motor technology even if it represented a potential cost saving opportunity. In terms of the Polya urn process, each simulation starts with the equal probability for each technology to be adopted $p\left(x_{0}\right)=50 \%$ [,i.e. the share of possible new adopters that have adopted a motor ship equals to the share of new adopters that have adopted a steam ship]. Further the process develops according to the probability function [6] entailing positive feedback to adoption.

Figure 5: Urn's cumulative growth rate in year $T_{i}$ as the percentage of the merchant fleet in the initial year $T_{0}$

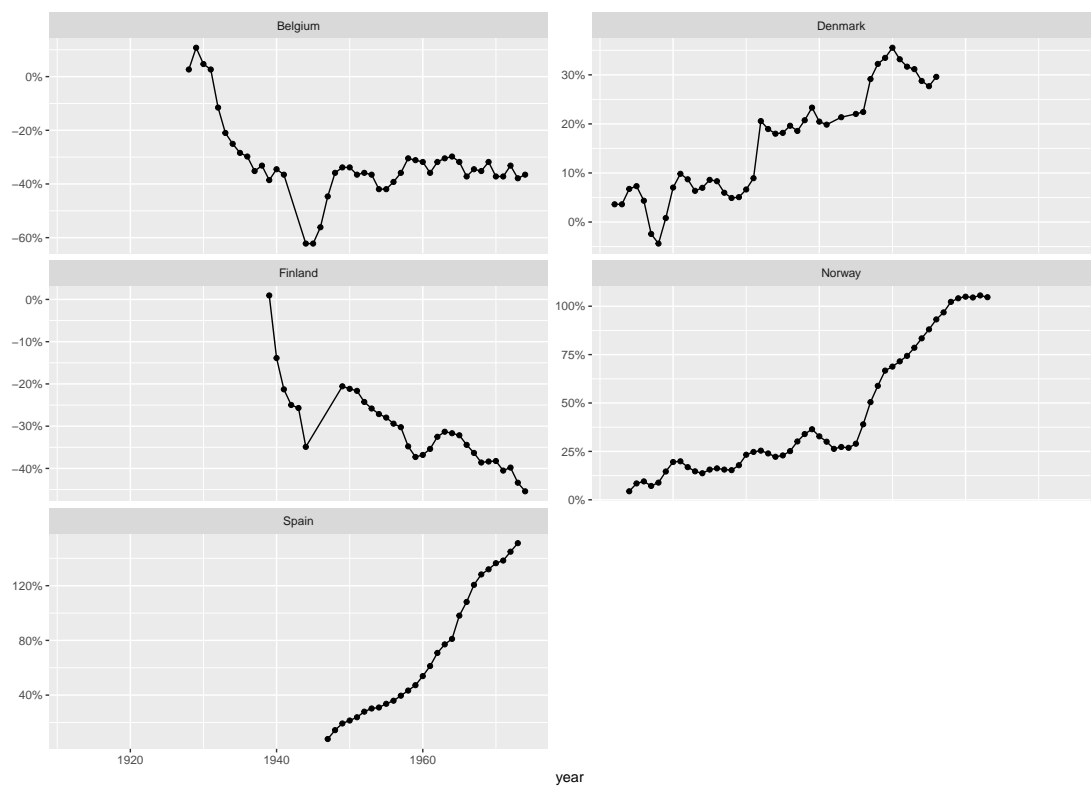

The number of balls in the urn corresponds to the size of the merchant fleet in a given year $T$. We assume that a ship is scrapped after 30 years of its exploitation. So every year we scrap $1 / 30 \approx 3 \%$ of the initial fleet stock. In terms of the Polya urn process, every year we remove the number of balls corresponding to the quantity of ships to be scrapped in the given year, we add the same number of new balls and we additionally add(remove) the number of balls corresponding to the difference in fleet stock between year $T$ and $T+1$. In this way the urn always matches the empirical data on the size of the merchant fleet in a given year, and we don't need to make additional assumptions to generate $n_{t}$ and $m_{t}$ time-series for our model (see equation [7]). Figure 5 shows urns' cumulative growth rates for the five

\footnotetext{
${ }^{2}$ This is a reasonable assumption to make in the absence of the micro-data on the preferences of initial adopters. However, the model can easily be adapted for any other distribution of preferences.
} 
countries in our dataset. We see that in Belgium and Finland the size of the fleet, i.e. the urn, decreased by $40 \%$ over the years in comparison with its initial size. In Spain, Norway and Denmark the fleet size raised by 200\%, 100\% and 30\%, respectively. The size of the fleet of a country and its dynamics affect the speed of diffusion. For example, the fleet of Belgium is 22 times smaller than the one of Norway. This contributes to explain why it took only 10 years to Belgium to arrive from $25 \%$ of the market share to $50 \%$, and it took to Norway - 18 years to do the same.

We keep track on the proportion of motor ships adoptions among new adoptions. This is our urn process described in the section 2 of the paper equation [7]. As one recalls, we start from the $50 \% 50$ proportion and every year this proportion path-dependently changes according to the realizations of the process. At the same time we keep track on the proportion of motor ships in the fleet of a country which changes every year lead by the decisions taken by new adopters in the given year. This process fully depends on the underlining urn process, and it imitates historical time series that we have. Formally,

$$
\overline{X_{T+1}}=\frac{\overline{n_{A}}+\sum_{t=1}^{T}\left(\sum_{i=1}^{n_{t}} \xi_{i}\left(X_{t}\right)-\sum_{j=1}^{m_{t}} \varepsilon_{j}\left(X_{t}\right)\right)}{\overline{n_{A}}+\overline{n_{B}}+\sum_{t=1}^{T}\left(n_{t}-m_{t}\right)}
$$

where $X_{t}$ evolves according to the urn process for the new adopters specified in the equation [7], while $\overline{n_{A}}$ and $\overline{n_{B}}$ are initial numbers of motor ships and steam ships in the fleet of a country, taken from the empirical data. We show that $\overline{X_{T+1}}$ mimics empirical time series on the market share of motor ships in a given country. We perform 80 simulations for each country. In half of the simulations the process leads to the market superiority of motor ships. On the Figure 6 we plot the average value of the market share in a given year across simulations ${ }^{3}$ and their dispersion together with historical times series. To test whether the simulated pattern is statistically different from the historical diffusion path, we fitted the logistic curve to the historical data and to the simulated data for each country and conducted $z$-test of statistical significance of the difference between the values of the regression coefficients of two logistic curves (Clogg et al., 1995; Paternoster et al., 1998). We did not find significant differences in all five countries.

\section{Conclusion}

Evolutionary models of technical change and diffusion find a natural representation in Polya urn as it is able to capture dynamic increasing returns and history dependence. However, so far the formulation has been mostly used to study the asymptotic properties of the process. Here we demonstrated that Polya urn can be fitted to the empirical data and that it may generate logistic curves of technology diffusion, thus producing trajectories generally observed in the adoptions of new technologies. As logistic processes are widespread in several domains of both social and natural sciences, this methodology will well find further applications to all those processes which entail self-reinforcing mechanisms of some kind.

\footnotetext{
${ }^{3}$ We consider only simulations that led to the market superiority of motor ships.
} 
Figure 6: Replication of the diffusion process with the Polya urn model

Black curves are historical time series.

Gray points indicate the average value of the market share across simulations of the model.

Gray bars indicate the dispersion of the market share across simulations of the model

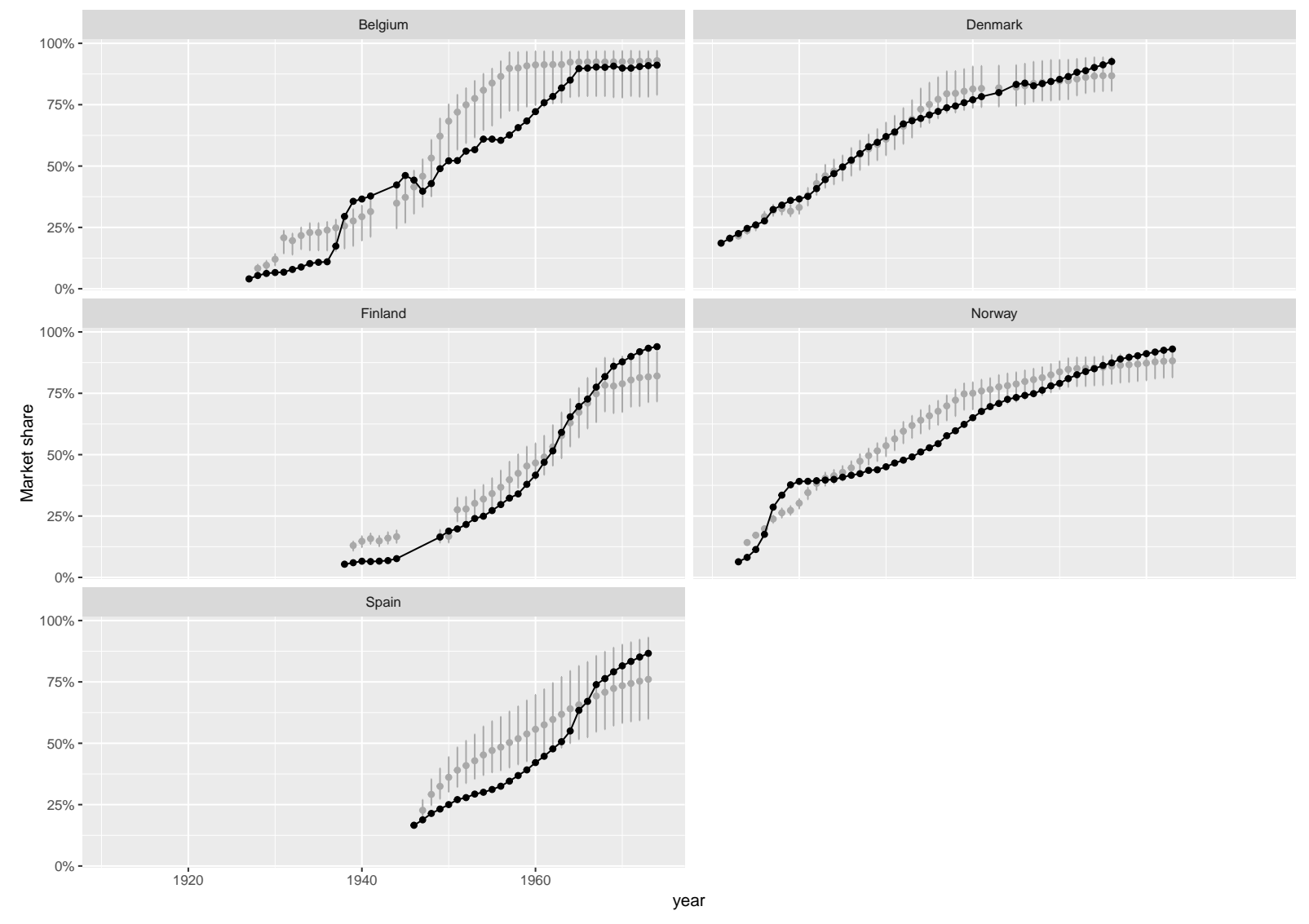




\section{References}

Arthur, W. B. (1989). Competing technologies, increasing returns, and lock-in by historical events. The Economic Journal 99(394), 116-131.

Arthur, W. B., Y. M. Ermoliev, and Y. M. Kaniovski (1986). Strong laws for a class of path-dependent stochastic processes with applications. In V. Arkin, A. Shiraev, and R. Wets (Eds.), Stochastic Optimization. Lecture Notes in Control and Information Sciences. Springer, Berlin, Heidelberg.

Arthur, W. B., Y. M. Ermoliev, and Y. M. Kaniovski (1987). Path-dependent processes and the emergence of macro-structure. European Journal of Operational Research 30(3), 294-303.

Bassanini, A. and G. Dosi (2006). Competing technologies, technological monopolies and the rate of convergence to a stable market structure. In C. Antonelli, D. Foray, B. H. Hall, and W. E. Steinmueller (Eds.), New Frontiers in the Economics of Innovation and New Technology. Essays in Honour of Paul A. David. Edward Elgar, Cheltenham.

Bottazzi, G. and A. Secchi (2003). A stochastic model of firm growth. Physica A: Statistical Mechanics and its Applications 324(1), 213-219.

Clogg, C. C., E. Petkova, and A. Haritou (1995). Statistical methods for comparing regression coefficients between models. American Journal of Sociology 100(5), 1261-1293.

Comin, D., B. Hobijn, and E. Rovito (2006). Five facts you need to know about technology diffusion. Technical report, National Bureau of Economic Research.

Cowan, R. (1990). Nuclear power reactors: a study in technological lock-in. The Journal of Economic History 50(03), 541-567.

David, P. A. (1985). Clio and the economics of QWERTY. The American Economic Review 75(2), $332-337$.

Davies, S. (1979). The Diffusion of Process Innovations. Cambridge University Press, Cambridge, UK.

Donnelly, P. (1986). Partition structures, polya urns, the ewens sampling formula, and the ages of alleles. Theoretical Population Biology 30(2), 271-288.

Dosi, G. (2000). The research on innovation diffusion: An assessment. In Innovation, Organization and Economic Dynamics: Selected Essays. Edward Elgar Publishing.

Dosi, G., Y. Ermoliev, and Y. Kaniovski (1994). Generalized urn schemes and technological dynamics. Journal of Mathematical Economics 23(1), 1-19.

Dosi, G., M. Grazzi, and D. Moschella (2015). Technology and costs in international competitiveness: from countries and sectors to firms. Research Policy 44(10), 1795-1814.

Dosi, G. and Y. Kaniovski (1994). On "badly behaved" dynamics: Some applications of generalized urn schemes to technological and economic change. Journal of Evolutionary Economics 4 (2), 93-123.

Griliches, Z. (1957). Hybrid corn: An exploration in the economics of technological change. Econometrica $25(4), 501-522$.

Hill, B. M., D. Lane, and W. Sudderth (1980). A strong law for some generalized urn processes. The Annals of Probability 8(2), 214-226.

Kaniovski, Y. M. and G. C. Pflug (1995). Non-standard limit theorems for Urn Models and stochastic approximation procedures. Communications in Statistics. Stochastic Models 11(1), 79-102. 
Kuba, M. and A. Panholzer (2012). Limiting distributions for a class of diminishing Urn Models. Advances in Applied Probability 44(01), 87-116.

Mahoney, J. (2000). Path dependence in historical sociology. Theory and Society 29(4), 507-548.

Mansfield, E. (1961). Technical change and the rate of imitation. Econometrica 29(4), 741-766.

Mitchell, B. (1998). International Historical Statistics: Europe 1750-1993. London: Palgrave Macmillan.

Nuvolari, A., B. Verspagen, and N. Von Tunzelmann (2011). The early diffusion of the steam engine in Britain, 1700-1800: a reappraisal. Cliometrica 5(3), 291-321.

Panholzer, A. and M. Kuba (2012). On death processes and Urn Models. In Proceedings of the 23rd International Meeting on Probabilistic, Combinatorial and Asymptotic Methods for the Analysis of Algorithms (AofA 2012).

Paternoster, R., R. Brame, P. Mazerolle, and A. Piquero (1998). Using the correct statistical test for the equality of regression coefficients. Criminology 36(4), 859-866.

Siegrist, K. (1987). An Urn Model with Bernoulli removals and independent additions. Stochastic Processes and Their Applications 25, 315-324.

Stapersma, D. (1996). 'Vulcanus versus Se-landia' or The early history of the marine diesel engine. Voorburg, The Netherlands.

Stoneman, P. and G. Battisti (2010). The diffusion of new technology. In B. H. Hall and N. Rosenberg (Eds.), Handbook of the Economics of Innovation. North-Holland.

Wilkins, H. (1925). Motorizing the ocean highways. Popular Mechanics Magazine 43(6), 956-961.

Windrum, P. (2004). Leveraging technological externalities in complex technologies: Microsofts exploitation of standards in the browser wars. Research Policy 33(3), 385-394.

Zhu, H. and F. Hu (2012). Interim analysis of clinical trials based on Urn Models. Canadian Journal of Statistics 40(3), 550-568. 